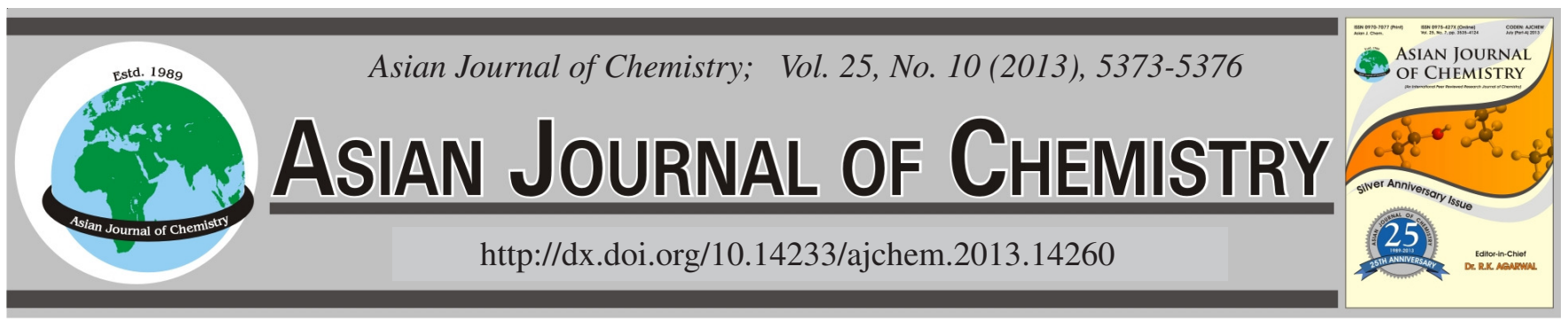

\title{
Detection of Organic Contaminants in Water Based on Polysulfone Microspheres with Hollow Core/Porous Shell Structure
}

\author{
XiAnHuA Li*, Yongcun GuO and Jun Zhang \\ School of Mechanical Engineering, Anhui University of Science and Technology, Huainan 232001, P.R. China \\ *Corresponding author: Fax: +86 554 6668934; Tel: +86 554 6313991; E-mail: xhli01@163.com
}

(Received: 30 June 2012;

Accepted: 8 April 2013)

AJC-13209

The monodisperse polysulfone microspheres with hollow core/porous shell structure were prepared using a water-in-oil-in-water
(W/O/W) emulsion solvent evaporation technique. The morphology could be controlled by varying the type of surfactant such as oleic
acid, poly(vinylpyrrolidone) and Tween 80 , respectively. The prepared microspheres are developed as reagent for the detection of
1-methyl-2-pyrrolidinone, which is based upon the morphology changes of microspheres to 1-methyl-2-pyrrolidinone. Results show
that the microspheres with hollow core/porous shell structure have an ultra sensitive response (as low as $10^{-7} \mathrm{M}$ ).

Key Words: Polysulfone microspheres, Hollow core/porous shell structure, Emulsion solvent evaporation technique, Detection of organic contaminants.

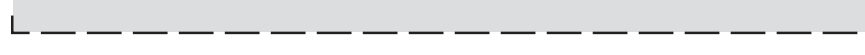

\section{INTRODUCTION}

Many solvents are potential threats to public health and the environment. As a promising detection method the physical methods ${ }^{1-3}$ include surface enhanced Raman spectroscopy, nuclear quadrupole resonance, energy-dispersive X-ray diffraction and neutron activation. These techniques are highly selective, but some are expensive and others are not easily fielded in a small, low-power package. Recently, organic polymers and optical fibers ${ }^{4}$ have been studied to detect vapours of explosive analytes. The transduction methods used include absorption, fluorescence, conductivity, etc. However, little attention was paid to the materials with special morphology, such as hollow core/porous shell structure for improvement in transduction method.

Hollow spherical materials have attracted a considerable amount of attention. Various synthetic approaches, such as hard and soft colloidal templating, layer-by-layer deposition, template-free synthesis and more recently microfluidics and particle-stabilized emulsions, have been developed to fabricate them. Recently, tailor-made hollow spheres, including tin oxide nanoparticle microshells ${ }^{5}$, silica hollow spheres and microballons $^{6}$, silica nanocages ${ }^{7}$ and poly(methyl methacrylate) microspheres ${ }^{8}$ with hollow core/porous shell structure, have been developed with these techniques. As a convenient procedure, the emulsion solvent evaporation technique has been reported extensively for the preparation of polymer microspheres. However, it has not been used to prepare porous mocrospheres.
Here we report the fabrication of polysulfone microspheres with hollow core/porous shell structure through the emulsion solvent evaporation technique. The morphology of microspheres can be changed when it is added into the 1-methyl-2-pyrrolidinone-water solution. Therefore, the polysulfone microspheres with special morphology are directly utilized as reagent for the detection of 1-methyl-2pyrrolidinone (NMP). The polysulfone microspheres with hollow core/porous shell structure have a higher sensitivity for the detection of 1-methyl-2-pyrrolidinone and the detection limit of 1-methyl-2-pyrrolidinone concentration reaches as low as $10^{-7} \mathrm{M}$.

\section{EXPERIMENTAL}

Polysulfone was provided by solvay advanced polymers. Poly(vinyl pyrollidone), poly(vinyl alcohol), oleic acid, Tween 80, methylene chloride (DCM) and 1-methyl-2-pyrrolidinone (NMP) were all analytical-grade reagents (Shanghai Chemical Reagent Co. Ltd., China). The other reagents were used as received without further purification. Deionized water was used throughout the experiment to prepare solution.

To prepare the polysulfone microspheres with hollow core/ porous shell structure, the oleic acid $(0.1 \mathrm{~g})$ was dissolved in 5 $\mathrm{mL}$ methylene chloride and then the $0.3 \mathrm{~mL}$ poly(vinyl alcohol) $(0.1 \%)$ solution was poured in to yield a pre-emulsion. The small internal aqueous droplets were surrounded by the mixture of surfactants, poly(vinyl alcohol) acting as a cosurfactant increased the efficiency of the interfacial activity between the 
oil and the aqueous phase. $0.1 \mathrm{~g}$ of polysulfone was dissolved in $1 \mathrm{~mL}$ of 1-methyl-2-pyrrolidinone and then slowly added into a pre-emulsion solution. This system was stirred for $2 \mathrm{~h}$ at room temperature. During this period, polysulfone tended to diffuse to the emulsion droplets. After this system was slowly added into $50 \mathrm{~mL}$ poly(vinyl alcohol) $(0.1 \%)$ solution for $2 \mathrm{~h}$ at $35^{\circ} \mathrm{C}$. The W/O/W emulsion was stabilized by the emulsion droplets and surfactants for outer aqueous phase. With shrinkage of the polysulfone shell caused by solvent evaporation, a hollow core formed. At the same time, it propelled the emulsion droplets to disengage themselves automatically from the polymeric shell, leaving behind a porous polymer shell. The synthesis materials were designated as PSF-1. The overall synthetic procedure is shown in Scheme-I. To understand the influence of the type of surfactant on the morphology, a set of experiments were performed with another two types of surfactant such as poly(vinyl pyrollidone) (PSF-2) and PSF-3, while the other synthetic parameters were kept constant.
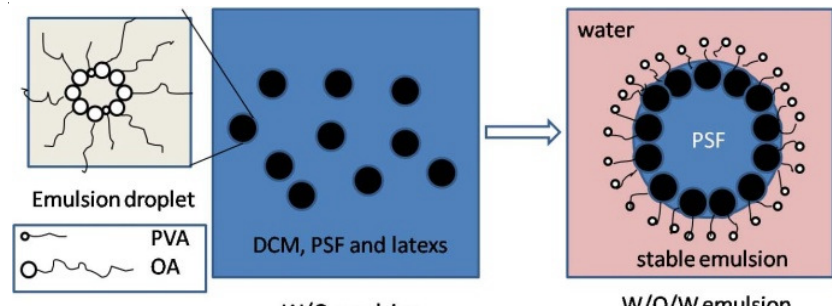

W/O emulsion
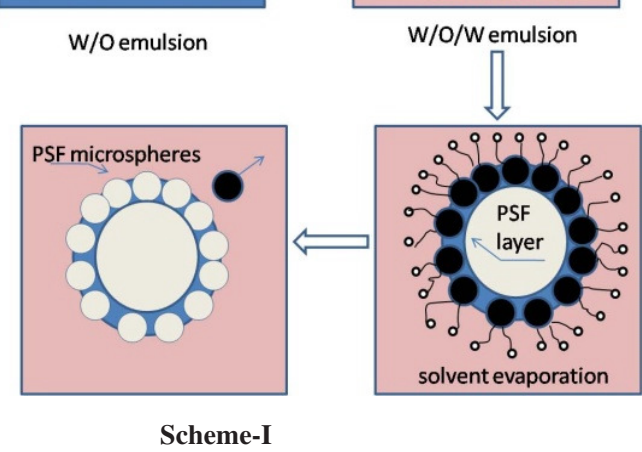

The detection of trace organic contaminants in water was carried out according to the procedure as follow. The microspheres were putted into mixed solvent (NMP/water or DMF/water) with the ration ranging from $1 / 100$ to $1 / 10^{7}$ and then separated and dried at vacuum.

Water contact angles (CAs) measurements were performed using an optical video contact angle instrument (model OCA 40, Dataphysics, Germany) at room temperature. Field emission scanning electron microscopy (FESEM) measurements were taken with a Hitachi S4800 scanning electron microscope. Transmission electronic microscopy was performed by using a JEM-2100F instrument with a field emission gun operating at $200 \mathrm{kV}$. Fourier transformation infrared spectra were obtained using a NEXUS-870 spectrophotometer.

\section{RESULTS AND DISCUSSION}

Fig. 1 shows the SEM and TEM images of the polysulfone microspheres fabricated by W/O/W. From Fig. 1a and b, we can see clearly that the microspheres are monodisperse with diameter ranging from 22 to $31 \mu \mathrm{m}$ when oleic acid was used as the surfactant. In addition, the shell of microspheres is porous with diameters in the range of 1-3 $\mu \mathrm{m}$. As shown in the inset of Fig. 1b, the polysulfone microspheres possess not only interconnected pores but also hollow core structure, observed from the broken microspheres. Fig. 1c and d depict the TEM images of the PSF-1 at the edge and center section. The results further indicated that the microspheres have hollow core/porous shell structure.
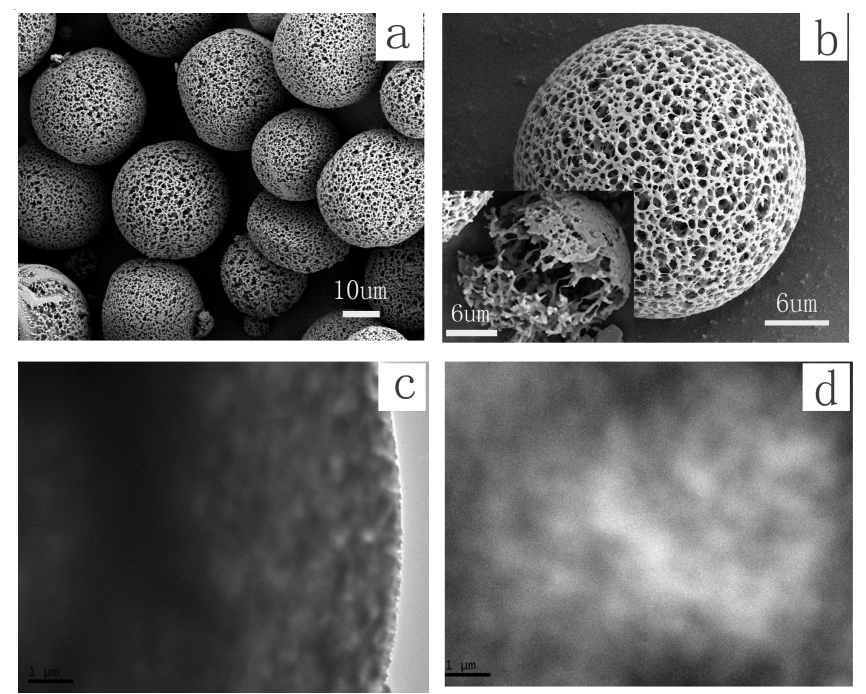

Fig. 1. SEM (a, b) and TEM (c, d) images of PSF-1 microspheres, inset shown in Figure $1 \mathrm{~b}$ is a broken PSF-1

It is well known that hydrophilic lypophilic balance number of a surfactant influence the emulsion stability. We try to investigate the effects of hydrophilic lypophilic balance numbers to the formation of microspheres. When poly(vinyl pyrollidone) with higher hydrophilic lypophilic balance number is used as surfactant, polysulfone microspheres (PSF-2) have smooth surface with few porous (Fig. 2a). Moreover, the polysulfone microspheres possess a decreased pore diameter of about $900 \mathrm{~nm}$ (Fig. 2b). When Tween 80 with the highest hydrophilic lypophilic balance number is used as surfactant, only a few polysulfone microspheres (PSF-3) are obtained (Fig. 2c) and the surface of the microspheres is rugged (Fig. 2d). The similar phenomena were observed in the preparation of poly(lactic-co-glycolic acid) (PLGA) microspheres ${ }^{9}$. The surface porosity could be controlled by different type of surfactant. The hollow core structure may be attributed to the stability of the surfactant. Nihant et al. ${ }^{10}$ found that the internal structure of microparticles could be changed from a multivesicular to a matrix-like structure depending on the stability of surfactant. It is obvious that the surfactant with different hydrophilic lypophilic balance numbers has significant impacts upon the morphology. In this study, a low hydrophilic lypophilic balance number surfactant (OA) contribute to the generation of polysulfone microspheres with hollow core/ porous shell structure. In addition, experiments find that the effects of temperature on the morphology are relatively small.

The spectrum of Fig. 3 reveals the bands at 1321 and 1188 $\mathrm{cm}^{-1}$, attributed to symmetric and asymmetric stretching vibrations of $\mathrm{S}=\mathrm{O}$ bonds in the polysulfone backbone. The band corresponding to the stretching vibration of C-O-C structures 
appears around $1264 \mathrm{~cm}^{-1}$. In addition, the sharp bands at 1620 , 1562 and $1403 \mathrm{~cm}^{-1}$ are attributed to benzene ring. The above results imply that the microspheres consist of polysulfone.
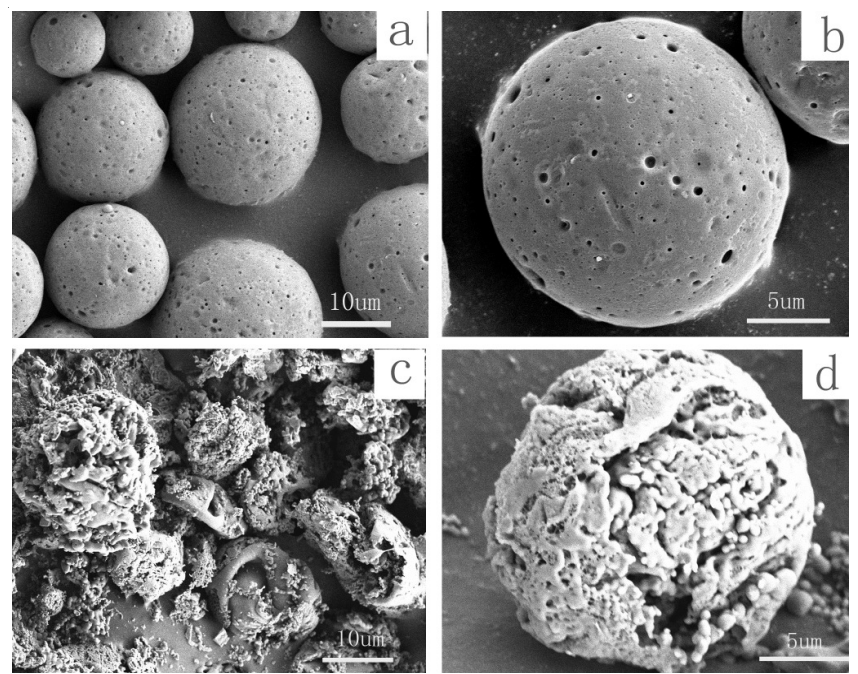

Fig. 2. SEM images of PSF-2 (a, b) and PSF-3 (c, d)

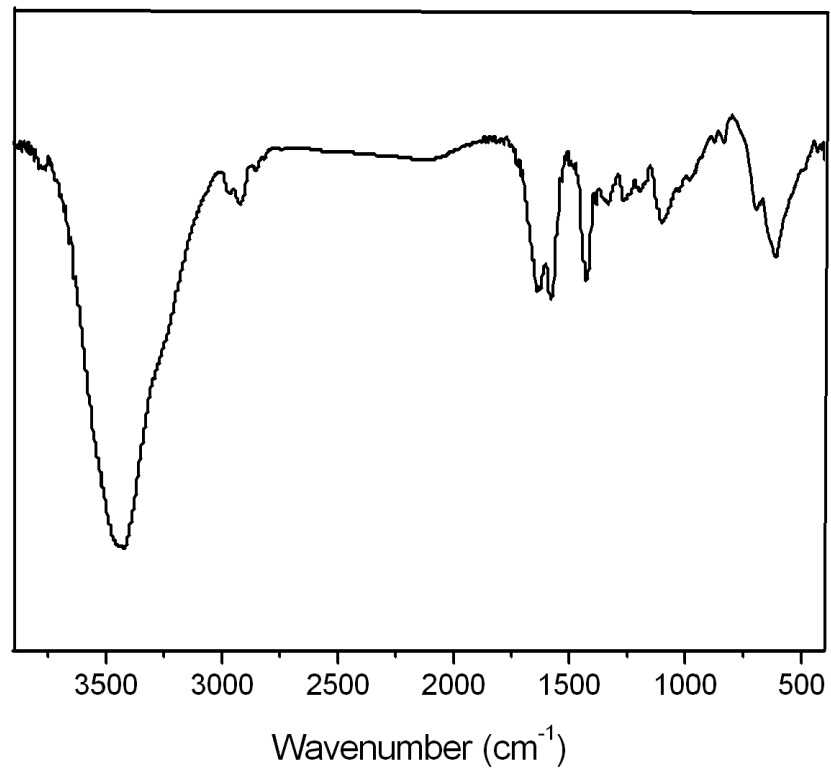

Fig. 3. FTIR spectra of polysulfone microspheres

Contact angle measurements show that the microspheres displayed superoleophilic properties. A drop of 1-methyl-2pyrrolidinone quickly spread on the bed of the polysulfone microspheres and the wetting time for a droplet of 1-methyl2-pyrrolidinone added to the microspheres was found to be $2 \mathrm{~s}$, as evidenced by dynamic contact angle measurements (Fig. $4 a-d)$.

Obviously, PSF-1 not only possesses clear surface structure in SEM but also easily absorb 1-methyl-2-pyrrolidinone . Thus, if 1-methyl-2-pyrrolidinone can be selectively absorbed into pore, the surface morphology must change in SEM. Fig. $5 \mathrm{a}, \mathrm{b}$ show the SEM images of PSF-1 obtained in the ratios of 1-methyl-2-pyrrolidinone and water from $1 / 100$ to $1 / 10^{7}$, respectively. When the ratio is $1 / 100$, the most of surface pores are dissolved to stick together compared with the inset (Fig. $5 a)$. When the ratio reduce to $1 / 10^{7}$, less pores are dissolved
(Fig. 5b). For comparison, the PSF-2 possessed special morphology is also studied. Result show that the morphology of PSF-2 is unchanged when the ratio of 1-methyl-2-pyrrolidinone and water is $1 / 100$ (Fig. 5c). These phenomena indicate that PSF-1 can detect trace 1-methyl-2-pyrrolidinone in water. This may contribute to the special morphology. The hollow core/ pore shell structure with the width of interconnected pores changing in 1-3 $\mu \mathrm{m}$ is favourable in 1-methyl-2-pyrrolidinone sorption. The same phenomena were observed by Zhu et al. ${ }^{11}$. High porosity and appropriate void size of sorbent provided large amounts of storage volume, which was the key for the high adsorption capacity. So, the morphology of PSF-1 has an ultra sensitive response to 1-methyl-2-pyrrolidinone.
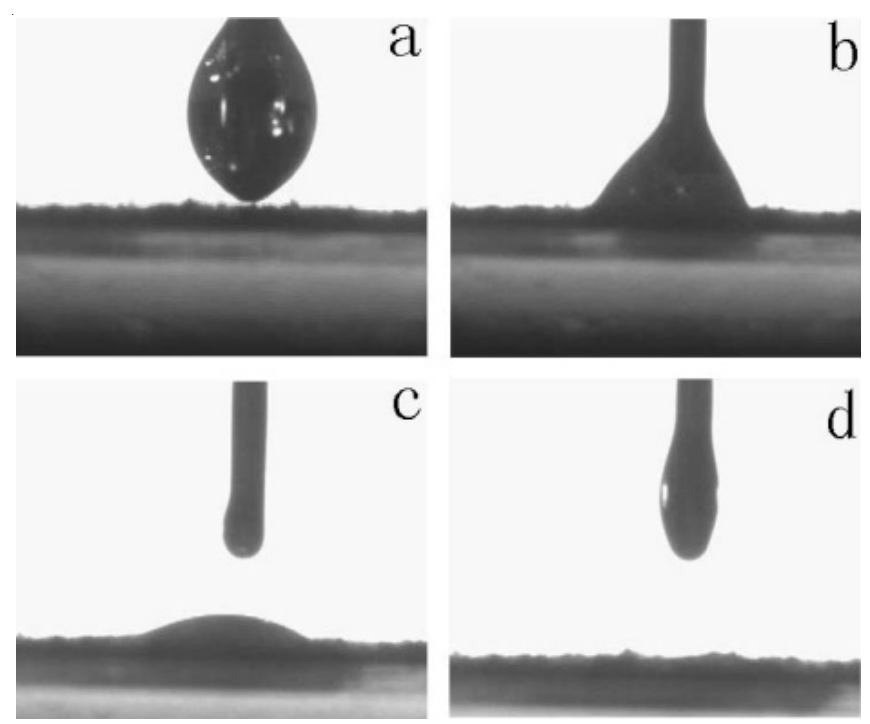

Fig. 4. (a-d) video snapshots of the wetting of an droplet of NMP on the bed of the PSF-1
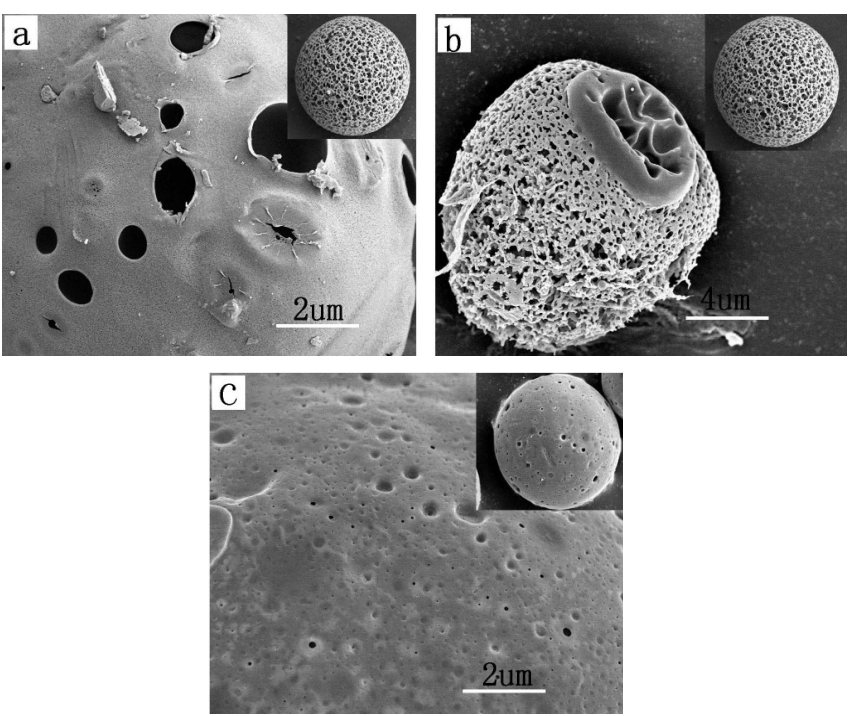

Fig. 5. SEM images of PSF microspheres obtained in the different ratios of NMP and water (a) 1/100 (PSF-1), (b) 1/107 (PSF-1), (c) 1/100 (PSF-2), inset is the original morphology of microspheres

\section{Conclusion}

In summary, the polysulfone microspheres with hollow core/porous shell structure have been prepared by the emulsion solvent evaporation technique. The special morphology have 
shown strong changes and been used here as transduction method to detect 1-methyl-2-pyrrolidinone. We believe that the special morphology detected by SEM would be a new class of transduction method allowing future practical applications in detection of organic contaminants.

\section{ACKNOWLEDGEMENTS}

This work is supported by the Youth and Shuobo Foundation of Anhui University of Science and Technology.

\section{REFERENCES}

1. J.M. Sylvia, J.A. Janni, J.D. Klein and K.M. Spencer, Anal. Chem., 72 , 5834 (2000)

2. G.V. Mozjoukhine, Appl. Magan. Reson., 4, 527 (2000).
3. R.D. Luggar, M.J. Farquharson, J.A. Horrocks and R.J. Lacey, X-Ray Spectrom., 27, 87 (1998).

4. V. Diz, M. Cassanello and R.M. Negri, Environ. Sci. Technol., 40, 6058 (2006).

5. C.J. Martinez, H. Bernard, C.B. Montgomery and S. Steve, Langmuir, 21, 7937 (2005).

6. B.C.I. Zoldesi and A. Imhof, Adv. Mater., 17, 924 (2005).

7. X.W. Lou, C. Yuan and Q. Zhang, Angew. Chem. Int. Ed., 45, 3825 (2006).

8. X.D. He, X.W. Ge, H.R. Liu, M.Z. Wang and Z.C. Zhang, Chem. Mater, 17, 5891 (2005).

9. C. Bouissou, U. Potter, H. Altroff, H. Mardon, V.D.W. Christopher, J. Control.. Rel., 95, 557 (2004).

10. N. Nihant, C. Schugens, C. Grandfils, J. Robert and T. Philippe, Pharm. Res., 11, 1479 (1994).

11. H.T. Zhu, S.S. Qiu, W. Jiang, D.X. Wu and C.Y. Zhang, Sci. Technol., 45, 4527 (2011). 